\title{
Removing Degeneracy of Microlensing Light Curves through Narrowband Photometry of Giants
}

\section{Citation}

Loeb, Abraham, and Dimitar Sasselov. 1995. "Removing Degeneracy of Microlensing Light Curves through Narrowband Photometry of Giants." The Astrophysical Journal 449 (1). https:// doi.org/10.1086/309625.

\section{Permanent link}

http://nrs.harvard.edu/urn-3:HUL.InstRepos:41393305

\section{Terms of Use}

This article was downloaded from Harvard University's DASH repository, and is made available under the terms and conditions applicable to Other Posted Material, as set forth at http:// nrs.harvard.edu/urn-3:HUL.InstRepos:dash.current.terms-of-use\#LAA

\section{Share Your Story}

The Harvard community has made this article openly available.

Please share how this access benefits you. Submit a story. 


\title{
REMOVING DEGENERACY OF MICROLENSING LIGHT CURVES THROUGH NARROWBAND PHOTOMETRY OF GIANTS
}

\author{
Abraham Loeb and Dimitar Sasselov ${ }^{1}$ \\ Harvard-Smithsonian Center for Astrophysics, 60 Garden Street, Cambridge, MA 02138 \\ Received 1995 April 3; accepted 1995 June 1
}

\begin{abstract}
The standard light curve of a microlensing event provides only two constraints on the five unknown parameters of the lens. We show that narrowband photometry during a microlensing event of a giant star can in addition determine the angular radius of the Einstein ring and the proper motion of the lens. This possibility results from the fact that the extended atmospheres of giants emit the cores of resonance lines primarily from a narrow ring (limb brightening). A considerable fraction of the clump giants in the bulge have a ring radius $\gtrsim 10^{12} \mathrm{~cm}$, within the range of Einstein radii for subsolar mass lenses. The extended thin ring also provides a sensitive probe of possible planetary companions as it sweeps across the lens plane. The ring signature can be detected photometrically, using a narrowband filter centered on resonance lines like Ca II $\lambda 3933$.

Subject headings: astrometry — dark matter — gravitational lensing
\end{abstract}

\section{INTRODUCTION}

By now there are more than 50 microlensing events detected toward the Large Magellanic Cloud (LMC) and the Galactic bulge (Alcock et al. 1995; Auborg et al. 1993; Udalski et al. 1994a). The standard light curve of an event is characterized by two observables: its peak amplitude and duration (Paczyński 1986). However, there are seven parameters that define the physical properties of the lens, namely its mass, its three velocity components, and its three position coordinates. The mass, distance, and transverse velocity of the lens combine to set the duration of the event, and together with the impact parameter they also define its peak amplitude. The two angular coordinates of the lens are also known. Breaking the degeneracy among the remaining lens parameters is of fundamental importance to the interpretation of microlensing light curves. For observations toward the LMC, it is essential to discriminate between lenses that are located within the LMC (Sahu 1994) and lenses which are part of the Galactic halo or thick disk (Gould, Miralda-Escudé, \& Bahcall 1994); for bulge observations, it is important to separate lenses that are embedded in the Galactic disk from lenses inside a possible Galactic bar (Zhao, Spergel, \& Rich 1995; Han \& Gould 1995). A variety of methods to partially remove the existing degeneracy were suggested recently. The most ambitious proposal involves a parallax satellite mission to measure the lens proper motion (Refsdal 1966; Gould 1992, 1994b, 1995a). Other proposals consider alterations of the standard light curve due to different properties of the source, such as its finite size (Gould 1994a, 1995b; Nemiroff \& Wickramasinghe 1994; Witt \& Mao 1994), its polarized emission (Simmons, Newsam, \& Willis 1995), spectral shifts due to its rotation and finite size (Maoz \& Gould 1994), or shifts in its color due to a luminous lens (Kamionkowski 1995; Udalski et al. 1994c). In this Letter, we show that narrowband photometry of lensed giant stars can be used to measure the proper motion and the angular radius of the Einstein ring of the lens.

Optical starlight is dominated by photospheric continuum

${ }^{1}$ Hubble Fellow. emission. The continuum emission generally peaks at the projected center of the stellar disk and falls-off toward the stellar limb. In cool stars, some spectral lines (such as $\mathrm{H} \alpha$ or Ca II) are still optically thick at the limb and therefore appear strongly in emission there. This resonant line scattering was predicted for the Sun in 1870 by C. A. Young and was later observed during solar eclipses (Zirin 1988). The limb brightening of various strong lines in the Sun extends only over a ring of width $\lesssim 0.01 R_{\odot}$. Cool stars with a much smaller surface gravity, such as giants and supergiants, have atmospheres which are more extended than the Sun by 1-2 orders of magnitude and therefore show a much stronger resonant line scattering. Limb brightening of supergiants has been observed directly only recently (Hannif et al. 1992).

For giant stars, the line emission ring has a radius of $0.05-1$ AU, comparable to the Einstein radius of subsolar mass $(\lesssim 0.1$ $M_{\odot}$ ) lenses. Such a ring is similar in size to the baseline of a microlensing parallax experiment by an Earth-satellite system. For a specific star, the actual radius of the ring can be measured up to a precision of $\sim 20 \%$ after the lensing event has ended, using traditional methods of high-resolution spectroscopy and infrared photometry (see McWilliam \& Rich 1994 for application to Galactic bulge giants). Based on the measured radius, it is then possible to determine the projected Einstein radius and the proper motion of the lens relative to the line of sight.

\section{NARROWBAND LIGHT CURVES OF GIANTS}

The amplification of a point source by a point mass, $M$, depends only on their projected separation d (Paczyński 1986),

$$
A(d)=\frac{d^{2}+2}{d\left(d^{2}+4\right)^{1 / 2}},
$$

where $d$ is expressed in units of the angular radius of the Einstein ring of the lens, $\theta_{E}=\left(\left[4 G M / c^{2}\right]\left[D_{\mathrm{LS}} / D_{\mathrm{OL}} D_{\mathrm{OS}}\right]\right)^{1 / 2}$, and $D_{\mathrm{OL}}, D_{\mathrm{LS}}$, and $D_{\mathrm{OS}}$ are the distances between the observer, lens and source. The total flux received from an extended source is therefore obtained by integration over its infinitesimal elements, 


$$
F(t)=\int_{0}^{\tilde{R}_{s}} r d r B(r) \int_{0}^{2 \pi} d \theta A(d),
$$

where $B(r)$ is the surface brightness profile of the source in the projected polar coordinates $(r, \theta)$ around its center. For simplicity, we assume that the stellar emission is circularly symmetric, and denote the source radius by $R_{s}=\widetilde{R}_{s} \times\left(\theta_{E} D_{\mathrm{OS}}\right)$. All projected length scales are normalized by the Einstein ring radius. Using the law of cosines

$$
d=\left|\mathbf{d}_{0}-\mathbf{r}\right|=\left(d_{0}^{2}-2 d_{0} r \cos \theta+r^{2}\right)^{1 / 2},
$$

where $d_{0}=\left(b^{2}+v^{2} t^{2}\right)^{1 / 2}$ is the projected separation between the lens and the source center, $b$ is the projected impact parameter of the source center, $v$ is the transverse velocity of the lens in units of $\theta_{E} D_{\mathrm{OL}}$, and $t=0$ is the midpoint time of the lensing event. For a circularly symmetric source, the resulting light curve is time-symmetric with $F(t)=F(-t)$.

The narrow-band light curve of a giant is obtained by substituting its center-to-limb intensity distribution, $B(r)$, in equation (2). In calculating $B(r)$ we ignore any surface features on the otherwise circularly symmetric stellar disk, i.e., our radiative transfer models are one-dimensional. As initial conditions for constructing spherical atmospheres we use constant-flux plane-parallel blanketed photospheres from Kurucz (1993), and outer atmosphere models from Dupree, Hartmann, \& Avrett (1984) and Harper (1992). The multilevel non-LTE radiative transfer in these models is then solved in a spherical geometry using the SMULTI code (Harper 1994). We performed calculations with several atomic models $(\mathrm{H}, \mathrm{Ca}$ II, Mg II) and found that both the $\mathrm{Ca}$ II $\mathrm{H}$ and $\mathrm{K}$ lines show significant scattering above the limb, and are well suited for photometry. The model used for the Ca II atom has six levels; it treats both resonance lines and the infrared triplet in detail. For other similar center-to-limb calculations see Sasselov \& Karovska (1994). An example for the most extended atmosphere we have considered is illustrated in Figure 1.

\section{GIANTS IN THE BULGE}

The giants in the bulge have a relatively high metalicity (McWilliam \& Rich 1994), implying a high opacity in their interiors. For a given stellar mass, a higher opacity favors convection and leads to a larger radius, $R_{s}$, and a cooler effective temperature (Iben \& Faulkner 1968). The red giants in the bulge are similar to the members of the old open cluster M67, whose color-magnitude relation is often used to represent the bulge (Ibata \& Gilmore 1995). The relation between absolute magnitude, color, and radius, can be established using direct measurements of giant radii in the solar neighborhood and through the calibration of the M67 cluster. We determine the extinction based on the better known value for $E(B-V)=0.48$ (e.g., McWilliam \& Rich 1994), and the transformations of Taylor (1986) for K2 III stars. We find $E(V-I)=0.65$ for the Cousins $V-I$ color. This result is in good agreement with the HST photometry of Baade's Window by Holtzman et al. (1993) — using the new abundance estimates by McWilliam \& Rich (1994), as well as with the recent result from W UMa systems by Rucinski (1995). Next, we use as most reliable the direct temperature calibration of $V-K$ photometry from lunar-occultation angular-diameters of field giants by Ridgway et al. (1980) and from Michelson interferometry by $\mathrm{Di}$ Benedetto \& Rabbia (1987) and Mo-

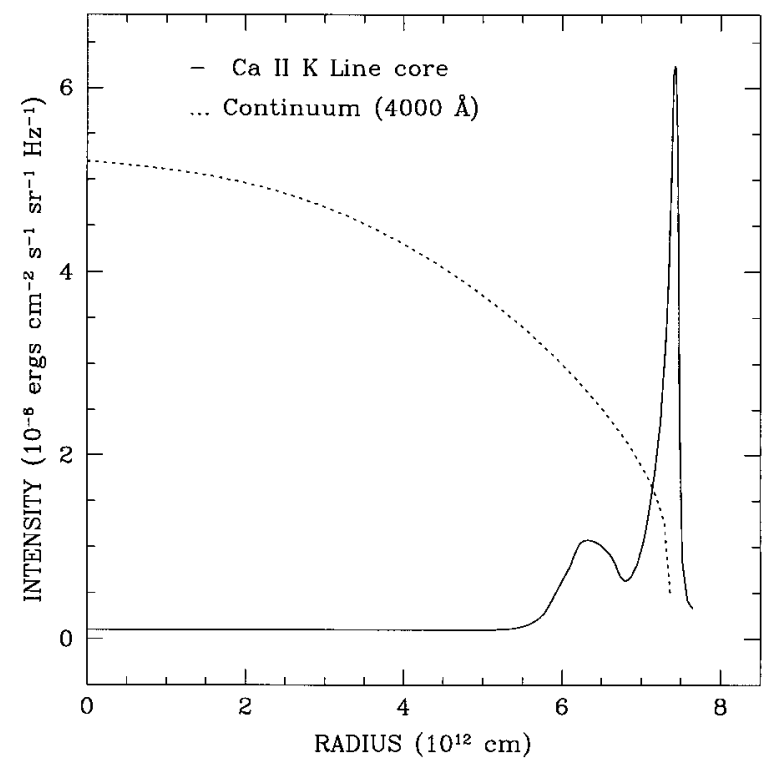

FIG. 1.-Stellar disk intensity distributions from a model atmosphere calculated in spherical geometry. The solid curve shows the center-to-limb intensity variation in the core of the $\mathrm{Ca}$ II $\mathrm{K} \lambda 3933$ line for a $100 R_{\odot} \operatorname{cool}(4150$ $\mathrm{K})$ giant star. The dotted curve shows the broad-band continuum intensity variation in the spectral region adjacent to the $\mathrm{Ca}$ II line. The intensity distribution for the $\mathrm{Ca}$ II $\mathrm{K}$ line core reflects our optimal choice of a wavelength band (see $\$ 4$ ).

zurkewich et al. (1991). This calibration is transformed to match the bulge giants by using the giants in M67 as surrogates with data from Taylor \& Joner (1988) and Joner \& Taylor (1988).

Using the above temperature calibration and adopting a distance to the Galactic center of $D_{\mathrm{OS}}=8 \mathrm{kpc}$, we have computed the radii of all stars for which the above relations are valid in the OGLE data set $(\sim 50 \%$ of the entire stellar population). Here we refer to the OGLE set with both $V$ - and I-band data (Stanek et al. 1994; see similar analysis by Witt 1995). Figure 2 shows the probability distribution of stellar radii for $\sim 90 \%$ of the OGLE stellar population $(V-I \geq 0.67)$. Since we are interested primarily in cool giants and supergiants, we did not use a separate radius calibration for dwarfs; the main sequence is shown in Figure 2 only to illustrate its relative fraction in the observed stellar population. We find that giants with radii $\gtrsim 20 R_{\odot}$ comprise $13 \%$ of the clump giants in Baade's Window. About $19 \%$ of all the stars have radii $\gtrsim 5 R_{\odot}$. The effect of a possible stellar bar on this distribution was found to be small and comparable to other uncertainties. However, since a significant fraction of the lenses may reside in such a bar (Zhao et al. 1995), we adopt a distance to the lens of $D_{\mathrm{OL}}=6 \mathrm{kpc}$ and a transverse lens velocity of $100 \mathrm{~km} \mathrm{~s}^{-1}$ in the numerical examples that follow.

\section{RESULTS}

The light curves of ringlike sources separate into two types: those with a single smooth peak and those with two sharp spikes. The first type is obtained when the lens is outside the ring trajectory, i.e. $b \gtrsim \widetilde{R}_{s}$, and the second type is obtained when the lens crosses the ring, i.e., $b<\widetilde{R}_{s}$. For the extreme first-type curves with $\widetilde{R}_{s} \ll b$, it is possible to solve equation (2) analytically. The amplification can then be Taylor expanded to second-order in $\left(d-d_{0}\right)$, where its derivatives are 


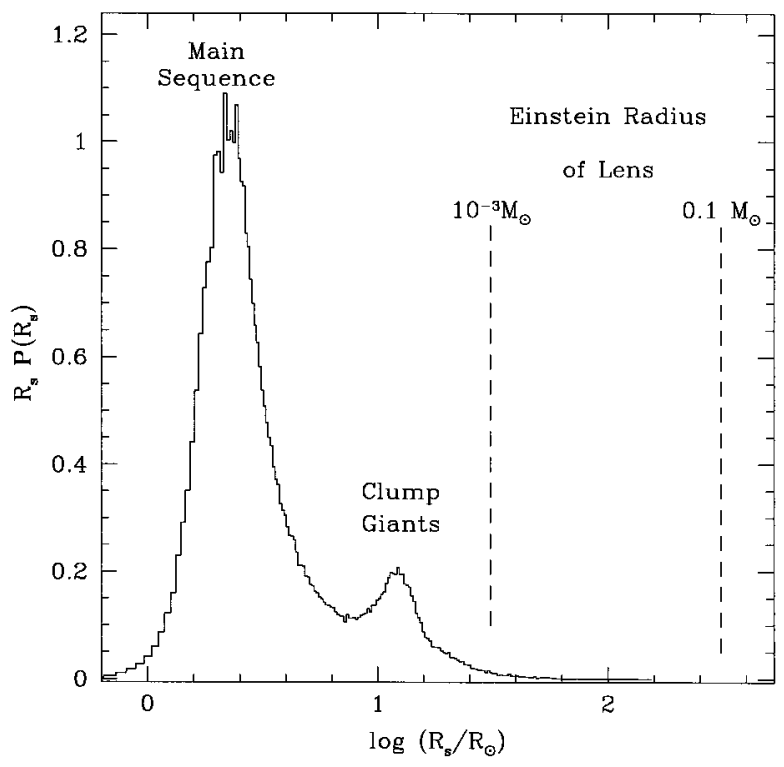

FIG. 2.-Probability distribution of stellar radii in Baade's Window, derived from the OGLE data set (Stanek et al. 1994). The clump giants are clearly separated from the main sequence. The Einstein radius for a lens mass of $10^{-3}$ $M_{\odot}\left(32 R_{\odot}\right)$ and a lens mass of $0.1 M_{\odot}\left(316 R_{\odot}\right)$ are marked, assuming $D_{\mathrm{OS}}=8$ $\mathrm{kpc}$ and $D_{\mathrm{OL}}=6 \mathrm{kpc}$. The main sequence is only qualitatively illustrated-our radius calibration applies to giants and supergiants.

obtained from equation (1) and the expression for $\left(d-d_{0}\right)$ from equation (3). The light curve of a point source, $F_{0}(t)$, is obtained by using $A\left(d_{0}\right)$ from equation (2). The fractional deviation from this light curve due to a source with a nonzero size is then

$$
\Delta(t) \equiv\left(F-F_{0}\right) / F_{0}=H(t)\left\langle r^{2}\right\rangle,
$$

where $\left\langle r^{2}\right\rangle \equiv\left[\begin{array}{llll}\int_{0}^{\tilde{R}_{s}} & r d r & B(r) & r^{2}\end{array}\right] /\left[\begin{array}{lll}\tilde{R}_{s} & r d r & B(r)\end{array}\right]$, and where $H(t) \equiv 8\left(d_{0}^{2}+1\right) d_{0}^{-2}\left(d_{0}^{2}+2\right)^{-1}\left(d_{0}^{2}+4\right)^{-2}$ is the temporal profile of the excess signal. For emission from a narrow ring, $\Delta(t)=H(t) \tilde{R}_{s}^{2}$. Thus, common events with $b \sim 0.5$ yield maximum values of $\Delta \sim \widetilde{R}_{s}^{2}$, so that a $3 \%$ accuracy in the photometry of the light curve which includes 10 points of observations can reveal the signature of a source radius $\widetilde{R}_{s} \gtrsim 0.1$. For our lens-source parameters, this threshold corresponds to a source radius $\gtrsim 2 \times 10^{12} \mathrm{~cm} \times\left(M / 0.1 M_{\odot}\right)^{1 / 2}$, which is in the expected range of radii for clump giants in the bulge (see Fig. 2). Based on a comparison to the exact solution, we find that equation (4) provides a good description to the ring light curve as long as $\widetilde{R}_{s}<b$.

However, the character of the light curve changes for $\tilde{R}_{s}>b$, when the ring is able to cross the lens. In this case, the event contains two pronounced peaks which are centered around the two crossing times of the ring through the lens. These crossing times are defined by the condition $d=\left|\mathbf{d}_{0}-\widetilde{\mathbf{R}}_{s}\right|=0$. The structure of the peaks depends on $\widetilde{R}_{s} ;$ a larger $\widetilde{R}_{s}$ makes the peaks narrower because the fraction of the ring area that is being affected by the lens gets smaller. By fitting the peak structure and separation to a stellar atmosphere model, it is possible to find both the impact parameter $b$ and the source size $R_{s}$ in units of the Einstein radius.

Figure 3 presents the distribution of narrowband light curves as a function of the impact parameter $b$. As this figure shows, it is much easier to identify the signal of the ring at small impact parameters. Figure 4 compares the narrowband

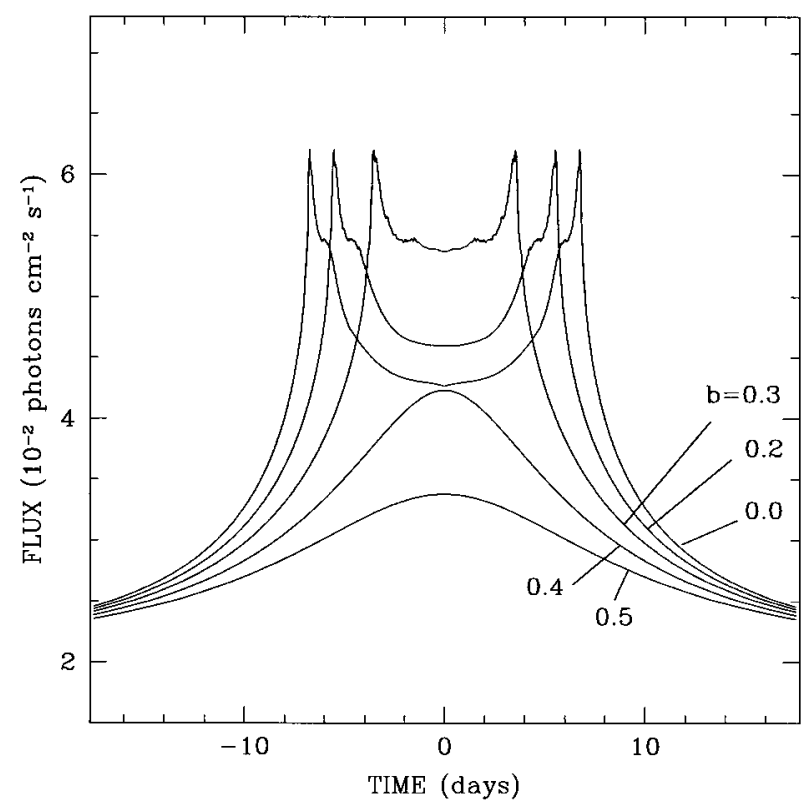

FIG. 3.-Flux amplification profiles in a narrow passband centered on the $\mathrm{Ca}$ II $\mathrm{K}$ line for lensing of the giant shown in Fig. 1 by a $0.1 M_{\odot}$ mass lens. The light curves are shown for different values of the dimensionless impact parameter, $b$. The radius of the Ca II ring of the giant is $\widetilde{R}_{s}=0.35$ in Einstein ring units. We plot the net photon flux on Earth for the spectrophotometry filter described in $\S 4$.

and the broadband light curves of the same source for a lens mass of $10^{-3} M_{\odot}$ and $b=0$. The main difference between the curves is the existence of two peaks in the narrowband curve. The significance of these peaks is emphasized by the similarity between the broadband curve (dashed curve) and a standard microlensing curve for a point source with some different

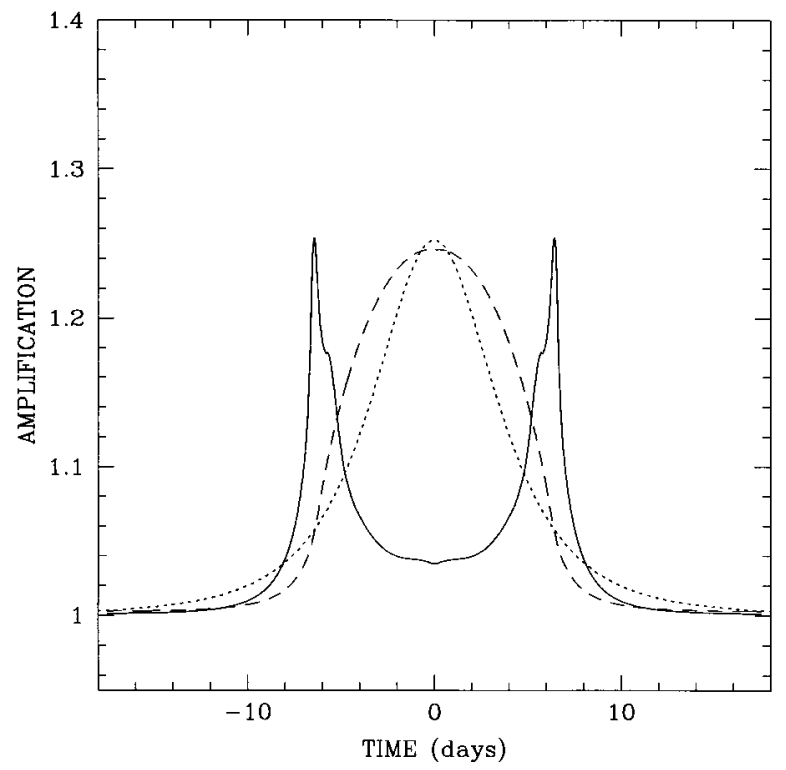

FIG. 4.-Comparison of light curves for a lens mass of $10^{-3} M_{\odot}$. The narrowband light curve (solid curve) is compared to the $4000 \AA$ broadband light curve (dashed curve), for the giant shown in Fig. 1 with $\widetilde{R}_{s}=3.5$ and $b=0$. The dotted curve is a standard microlensing curve for a point source, chosen to fit closely the broadband curve; it corresponds to $b=1.15$ and a transverse lens velocity of $50 \mathrm{~km} \mathrm{~s}^{-1}$. 
impact parameter and velocity (dotted curve). Although the small difference between these light curves is detectable, the extraction of the value of $\widetilde{R}_{s}$ from this difference could be complicated by observational errors or blending with unlensed starlight in crowded fields (Di Stefano \& Esin 1995). The finite source signature can be measured more reliably and unambiguously from the two peaks in its narrowband light curve. For $D_{\mathrm{OL}}=6 \mathrm{kpc}$, the fraction of giant lensing events with $b<\widetilde{R}_{s}$ is of order $\left\langle\widetilde{R}_{s}\right\rangle \approx 4 \% \times\left(M / 0.1 M_{\odot}\right)^{-1 / 2}$ (see Fig. 2$)$.

In practice, for the narrowband photometry required here one could use a modern version of the four-channel photoncounting spectrophotometer (HKP2) of the Mount Wilson HK project (Duncan et al. 1991) with $20 \AA$ wide reference bands and $2 \AA$ wide $\mathrm{H}$ and $\mathrm{K}$ bands. The narrow bands are positioned for each star according to the Earth's orbital motion and the velocity of the star; the latter can easily be obtained spectroscopically to an accuracy of $\sim 20 \mathrm{~km} \mathrm{~s}^{-1}$. For a giant with $R=20 R_{\odot}$ and $T_{\text {eff }}=4400 \mathrm{~K}$, the net flux in these $\mathrm{H}$ and $\mathrm{K}$ passbands is $4 \times 10^{5}$ ergs $\mathrm{cm}^{-2} \mathrm{~s}^{-1}$ at the stellar surface (see Rutten 1984 for absolute flux calibration in the passbands). With the adopted distance and extinction to the bulge, this translates to a detection rate of about 1 photon $\mathrm{s}^{-1}$ on a 2 meter telescope. Therefore, high quality ( $\pm 2 \%)$ narrowband photometry can be achieved using the HKP2 instrument with an integration time of $\sim 45\left(D_{t} / 2 \mathrm{~m}\right)^{-2} A^{-2}$ minutes on a telescope with a diameter $D_{t}$, where $A$ is the amplification. In addition, giants with $R_{s}>20 R_{\odot}$ require much shorter integration times as can be seen from Figure 3. Occasionally, a microlensing event may involve a chromospherically active giant which happens to have an active phase at the time of the event. Such activity could distort the circular symmetry of the emission ring or add bright spots on the stellar disk. However, subsequent photometry would be able to determine whether the lensed star was likely to have been active during the event.

\section{DISCUSSION}

A considerable fraction of the giants in the bulge have a radius $R_{s} \gtrsim 10^{12} \mathrm{~cm}$ (see Fig. 2). As illustrated in Figure 1, the narrowband (resonance line) images of these giants are thin rings. When a ringlike image crosses a lens, its time-symmetric light curve shows two sharp peaks. These narrow peaks provide an unambiguous signature of the finite source size.
The broadband light curve contains a smooth, weaker signature of the source size (see Fig. 4), that could be inaccurately interpreted. The spectroscopically measured radius can later be combined with the narrowband light curve to infer the proper motion and the angular radius of the Einstein ring of the lens.

The existence of an extended thin emission ring enhances the probability of detecting low-mass companions of the lens, such as planets. First, the finite source size sweeps through a wide strip in the lens-companion plane. Thus, the probability of intersecting the lensing region of influence of a companion planet is increased relative to the case of a point source by the ratio between the source size $R_{s}$ and the Einstein radius of the planet. For bulge observations, this ratio is $\sim\left(R_{s} / 2 \times 10^{11} M_{\mathrm{pl}}^{1 / 2}\right.$ $\mathrm{cm})$, where $M_{\mathrm{pl}}$ is the planet mass in earth mass units. Second, the planetary amplification is not smoothed over time by the entire stellar disk as in the case of broadband observations, but only by the width of the narrow emission ring-which is an order of magnitude smaller (see Fig. 4). Even in the extreme case of Fig. 1, where $R_{s}=100 R_{\odot}$, the width of the emission ring is still smaller than the Einstein radius of a Jupiter-mass planet $\sim 2 \times 10^{12} \mathrm{~cm}$. The ring provides two chances for the detection of a planetary-crossing signature. Based on the timing of these crossings relative to the primary lens crossings, it is possible to infer both the angular separation and the orientation of the companion relative to the primary lens. The proper motion of the two objects can be compared for any difference associated with their relative orbital motion. Each planetary crossing signature may be multiply peaked and is expected to last $\lesssim 1$ day (see Gould \& Loeb 1992). Once a lensing event of a giant star has triggered one of the currently operating early-warning systems (e.g., Udalski et al. 1994b), it would be useful to monitor its light curve on an hourly basis with a narrowband filter. This may prove to be an efficient method in searching for planetary systems around stellar lenses.

We thank R. Di Stefano, A. Gould, G. Harper, and S. Mao for useful discussions. D. D. S. acknowledges support for this work by NASA and STScI through Hubble Fellowship grant HF-1050.01-93A.
Alcock, C., et al. 1995, ApJ, 445, 133

Auborg, E., et al. 1993, Nature, 365, 623

Di Benedetto, G. P., \& Rabbia, Y. 1987, A\&A, 188, 114

Di Stefano, R., \& Esin, A. A. 1995, ApJ, 448, L199

Duncan, D. K., et al. 1991, ApJS, 76, 383

Dupree, A. K., Hartmann, L., \& Avrett, E. H. 1984, ApJ, 281, L37

Gould, A. 1992, ApJ, 392, 442 1994a, ApJ, 421, L71

1994b, ApJ, 421, L75

$1994 b$, ApJ, 421, L75
$1995 \mathrm{a}$, ApJ, 441, L21 1995b, ApJ, 441, 77

Gould, A., \& Loeb, A. 1992, ApJ, 396, 104

Gould, A. Miralda-Escudé, J., \& Bahcall, J. N. 1994, ApJ, 423, L105

Han, C., \& Gould, A. 1995, ApJ, 447, 53

Hannif, C. A., Ghez, A. M., Gorham, P. W., Kulkarni, S. R., Matthews, K., \&

Neugebauer, G. 1992, AJ, 103, 1662

Harper, G. M. 1992, MNRAS, 256, 37

. 1994, MNRAS, 268, 894

Holtzman, J. A., et al. 1993, AJ, 106, 1826

Ibata, R., \& Gilmore, G. 1995, MNRAS, submitted

Iben, I., \& Faulkner, J. 1968, ApJ, 153, 101

Joner, M. D., \& Taylor, B. J. 1988, AJ, 96, 218

Kamionkowski, M. 1995, ApJ, 442, L9

Kurucz, R. 1993, in Light Curve Modeling of Eclipsing Binary Stars, ed. E. F. Milone (New York: Springer), 93

\section{REFERENCES}

Maoz, D., \& Gould, A. 1994, ApJ, 425, L67

McWilliam, A., \& Rich, R. M. 1994, ApJS, 91, 749

Mozurkewich, D et al 1991, AJ, 101, 2207

Nemiroff, R. J., \& Wickramasinghe, W. A. D. T. 1994, ApJ, 424, L21

Paczyński, B. 1986, ApJ, 304, 1

Refsdal, S. 1966, MNRAS, 134, 315

Ridgway, S. T., Joyce, R. R., White, N. M., \& Wing, R. F. 1980, ApJ, 235, 126 Rucinski, S. 1995, ApJ, 446, L19

Rutten, R. G. M. 1984, A\&A, 130, 353

Sahu, K. 1994, Nature, 370, 275

Sasselov, D. D., \& Karovska, M. 1994, ApJ, 432, 367

Simmons, J. F. L., Newsam, A. M., \& Willis, J. P. 1995, MNRAS, submitted

Stanek, K. Z., et al. 1994, ApJ, 429, L73

Taylor, B. J. 1986, ApJS, 60, 577

Taylor, B. J., \& Joner, M. D. 1988, AJ, 96, 211

Witt, H. J. 1995, ApJ, 449, in press

Witt, H. J., \& Mao, S. 1994, ApJ, 430, 505

Udalski, A. et al. 1994a, Acta Astron., 44, 165

1994b, Acta Astron., 44, 227

1994c, ApJ, 436, L103

Zhao, H. S., Spergel, D. N., \& Rich, R. M. 1995, ApJ, 440, L13

Zirin, H. 1988, Astrophysics of the Sun (Cambridge: Cambridge Univ. Press),

161-162 